KEK-TH-1493, J-PARC-TH-0001

\title{
Test of CDF dijet anomaly within the standard model
}

\author{
H. Kawamura, ${ }^{1}$ S. Kumano, ${ }^{1,2}$ and Y. Kurihara ${ }^{1}$ \\ ${ }^{1}$ Institute of Particle and Nuclear Studies \\ High Energy Accelerator Research Organization (KEK) \\ 1-1, Ooho, Tsukuba, Ibaraki, 305-0801, Japan \\ 2 J-PARC Branch, KEK Theory Center, Institute of Particle and Nuclear Studies, KEK \\ and Theory Group, Particle and Nuclear Physics Division, J-PARC Center \\ 203-1, Shirakata, Tokai, Ibaraki, 319-1106, Japan
}

(Dated: October 27, 2011)

\begin{abstract}
Dijet anomaly reported by the CDF (Collider Detector at Fermilab) collaboration in $1.96 \mathrm{TeV} p \bar{p}$ collisions is investigated within the standard model by considering effects of parton distribution functions on various processes: $W+\operatorname{dijet,~} Z+\operatorname{dijet,} W W, Z W$, and top production. Since the anomalous peak exists in the dijet-mass region of $140 \mathrm{GeV}$ with the $p \bar{p}$ center-of-mass energy $\sqrt{s}=1.96 \mathrm{TeV}$, a relevant momentum fraction $x$ of partons is roughly 0.1 . In this $x$ region, recent HERMES semiinclusive charged-lepton scattering experiment indicated that the strange-quark distribution could be very different from a conventional one, which has been used for many years, based on oppositesign dimuon measurements in neutrino-induced deep inelastic scattering. We investigated effects of such variations in the strange-quark distribution $s(x)$ on the anomaly. We found that distributions of $W+$ dijets and other process are affected by the strange-quark modifications in wide dijet-mass regions including the $140 \mathrm{GeV}$ one. Since the CDF anomaly was observed in the shoulder region of the dijet-mass distribution, a slight modification of the distribution shape could explain at least partially the CDF excess. Therefore, it is important to consider such effects within the standard model for judging whether the CDF anomaly indicates new physics beyond the standard model. We also show modification effects of the strange-quark distribution in the LHC (Large Hadron Collider) kinematics, where cross sections are sensitive to a smaller- $x$ region of $s(x)$.
\end{abstract}

PACS numbers: 13.85.-t, 13.87.-a, 12.38.-t

\section{INTRODUCTION}

A signature beyond the standard model has been searched by the Tevatron at Fermilab (Fermi National Accelerator Laboratory) and by the LHC (Large Hadron Collider) at CERN (European Organization for Nuclear Research). So far, no explicit signature has been found yet. However, the CDF (Collider Detector at Fermilab) collaboration reported a possible discovery of new phenomenon in high- $p_{T}$-lepton plus dijet production with missing $E_{T}$ in $p \bar{p}$ collisions at the center-of-mass energy of $1.96 \mathrm{TeV}[1]$. They showed the $W+$ dijet cross section and other possible contributions as a function of the dijet mass $m_{j j}$. Their data indicated an anomalous peak in the region $m_{j j}=140 \mathrm{GeV}$. It is anomalous in the sense that such an excess is difficult to be explained within the standard model. On the other hand, such a peak cannot be found in another Tevatron experiment D0 [2], so that it may take time to settle down this issue experimentally.

We may recollect that there was an issue of jet-cross section excess in a CDF experiment of 1996 [3]. Jetproduction cross sections were measured in the $p \bar{p}$ collisions, and the data were shown as a function of jet transverse energy $\left(E_{T}\right)$. Since the cross sections were significantly larger than the QCD predications in the energy region $E_{T} \sim 400 \mathrm{GeV}$, the excess used to be considered as a signature beyond the standard model. However, it turned out that the jet excess could be explained if the gluon distribution is modified at large $x(\sim 0.6)$ [4], where $x$ is the momentum fraction of a gluon in the nucleon.
For explaining the new CDF data of 2011, interesting theoretical ideas have been proposed [5] mostly by models beyond the standard model. The ideas include new $Z^{\prime}$ boson, supersymmetry, Higgs boson, relation to string theory, and so on. However, the experience on the CDFjet issue of 1996 indicates that careful studies should be done within the standard model, particularly by estimating effects of parton distribution functions (PDFs).

A fraction of the proton or antiproton beam energy $980 \mathrm{GeV}(=1.96 \mathrm{TeV} / 2)$ needs to be transferred to one of the dijets with the energy about $70 \mathrm{GeV}(=140 \mathrm{GeV}$ /2). Therefore, the relevant region of the momentum fraction $x$ for initial partons is $x \sim 0.1$. This is a very rough estimate because some of the beam momentum should be carried, for example, by $W$ in the final state. We need to check whether the PDFs are well determined in this $x$ region in order to warrant the possibility that the CDF result is a new discovery beyond the standard model.

The PDFs of the nucleon have been investigated for many years, and they may be considered to be established except for extreme kinematical conditions. In fact, the valence-quark distributions are rather well determined due to abundant charged-lepton deep-inelastic-scattering (DIS) data together with neutrino DIS ones by using strong constraints of charge and baryon-number conservations. There is a recent issue of a nuclear modification difference between the charged-lepton and neutrino reactions [6]. It affects the determination of valence-quark distributions because neutrino-nucleus DIS data are used 
for extracting "nucleonic" PDFs. However, it should not be large enough to affect the CDF anomaly.

On the other hand, antiquark distributions are not well determined, particularly for strange and heavy quarks. The $\bar{u}$ and $\bar{d}$ distributions also have some uncertainties; however, they are relatively well determined in the region $0.05<x<0.2$ due to Drell-Yan experiments at Fermilab in combination with DIS measurements [7]. Furthermore, a new Drell-Yan experiment is currently in progress at Fermilab [8], and it could be continued at other hadron facilities such as the J-PARC (Japan Proton Accelerator Research Complex) [9]. The gluon distribution function $g(x)$ played an important role in explaining the CDF jet result in 1996 [4]. However, it may not be a significant contribution in the present case because $g(x)$ is constrained by scaling-violation measurements of the HERA (Hadron-Electron Ring Accelerator) except for the distribution in the large- $x$ region. Therefore, the first parton distribution, which we need to consider in connection with the new CDF anomaly, is the strange-quark distribution.

The strange-quark distribution $s(x)$ may have been thought to be determined from the opposite-sign dimuon measurements in neutrino-induced DIS. Current situation is summarized in Ref. [10]. There are some variations among experimental groups; however, $\int d x x(s+\bar{s})$ is $30-50 \%$ of the light-antiquark distribution $\int d x x(\bar{u}+$ $\bar{d})$. By considering the neutrino measurements, all the global PDF analysis had been done by assuming that $s(x)+\bar{s}(x)$ has the same $x$-dependence with $\bar{u}(x)+\bar{d}(x)$ until recently. There are some recent attempts to assign more flexible functional forms which are independent of $\bar{u}(x)+\bar{d}(x)$ [11]. However, the strange-quark distribution is not accurately constrained as typically shown by large uncertainties of $s(x)$ by the CT10 parametrization [11].

In 2008, the HERMES collaboration cast doubt on the conventional strange-quark distribution [12]. They measured semi-inclusive hadron production in charged-lepton DIS and they obtained a very different strange-quark distribution from the ones by global analyses with the neutrino DIS data. The distribution $s(x)$ is much softer than usual global-analysis distributions at $Q^{2}=2.5 \mathrm{GeV}^{2}$. For example, the $s(x)$ is two-times larger than the usual one at $x=0.03$ and it is much smaller at $x=0.2$. Of course, hadron-production analysis depend much on the employed fragmentation functions, which have large uncertainties for kaon functions [13]. Such an ambiguity needs to be considered in evaluating the HERMES result seriously.

The light antiquark distributions $\bar{u}(x), \bar{d}(x)$, and $\bar{s}(x)$ are considered to be equal in perturbative QCD (pQCD) because they are produced from the gluon splitting process $g \rightarrow q \bar{q}$ and their masses are expected to be negligible in comparison with a hard scale of a reaction. Of course, higher-order $\mathrm{pQCD}$ corrections could give rise to differences between $\bar{u}, \bar{d}$, and $\bar{s}$ because of $Q^{2}$ evolution with a mixing term $q \leftrightarrow \bar{q}$ in a splitting function at the next-toleading order (NLO) together with differences between the initial distributions $u, d$, and $s$ [7]. However, such NLO effects are not large enough to explain the measured differences, so that a non-perturbative mechanism is needed for explaining the differences between $\bar{u}, \bar{d}$, and $\bar{s}$. For the details of these non-perturbative models, the reader may look at Ref. 7].

It is the purpose of this article to discuss whether variations of $s(x)$, such as the one suggested by the HERMES, could affect the CDF anomaly. In particular, cross sections of $W+$ dijet, $Z+\operatorname{dijet,~} W W, Z W$, and top production processes are calculated by using the code GR@PPA (GRace At Proton-Proton/Antiproton collisions) [14] by taking three functional forms of $s(x)$. The GR@PPA is an event generator for $V(W$ and $Z)+$ jets, $V V$, and $\mathrm{QCD}$ multi-jet production processes in $p p$ and $p \bar{p}$ collisions.

This article is organized in the following way. In Sec. II. our method is explained for calculating the cross sections by the GR@PPA, and employed strange-quark distributions are explained in Sec. III. Results for the lepton-plus-dijet cross sections are shown in Sec. IV] and they are summarized in Sec. V.

\section{METHOD FOR CALCULATING CROSS SECTIONS}

In describing the cross sections for $W+$ dijet, $Z+$ dijet, $W W, Z W$, and top productions, the event generator GR@PPA [14] is used in this work. First, this event generator is explained in Sec. IIA. We use the CTEQ6L1 distributions for the PDFs in the proton and anti-proton. Next, in order to find effects of the strange-quark distribution, different forms of $s(x)$ are provided in the GR@PPA code as explained in Sec. III The other PDFs are not modified from the CTEQ ones.

\section{A. Event generator GR@PPA}

The GR@PPA is a framework to calculate cross sections of proton-proton/antiproton collisions by using the GRACE [15], in which Feynman diagrams are automatically computed at the one-loop level as well as the tree level in the standard model. The GRACE is intended mainly for lepton collisions, so that it is implemented by including features of hadronic collisions such as the PDFs in the GR@PPA [14].

The cross section for a certain hadron-hadron reaction process can be calculated by the sum of partonic subprocess contributions

$$
\sigma=\sum_{i, j, F} \int d x_{1} d x_{2} d \hat{\Phi}_{F} f_{i}^{1}\left(x_{1}, Q^{2}\right) f_{j}^{2}\left(x_{2}, Q^{2}\right) \frac{d \hat{\sigma}_{i j \rightarrow F}(\hat{s})}{d \hat{\Phi}_{F}}
$$

where $f_{i}^{h}\left(x_{h}, Q^{2}\right)$ is the PDF of the hadron $h, x_{h}$ is the momentum fraction carried by the parton $i$ or $j, Q^{2}$ is a hard scale in the reaction, and $d \hat{\sigma}_{i j \rightarrow F}(\hat{s}) / d \hat{\Phi}_{F}$ is the cross section for the partonic subprocess $i+j \rightarrow F$. Here, 
$i$ and $j$ indicate parton types, $F$ is the final state, $\hat{s}$ is the square of the center-of-mass energy in the parton level $\left(\hat{s}=\left(p_{i}+p_{j}\right)^{2}\right)$, and $\hat{\Phi}_{F}$ is the phase space of the final state.

As for the partons, we take into account 11 types $(u$, $d, s, c, b, g, \bar{u}, \bar{d}, \bar{s}, \bar{c}$, and $\bar{b})$. There are a huge number of subprocesses which contribute to the cross section. It is obvious that some of them have identical diagrammatic structure with slight changes in coupling constants such as the CKM (Cabibbo-Kobayashi-Masukawa) factors. Therefore, computation time is significantly reduced by calculating selected base-subprocesses and then weight factors are simply multiplied:

$$
\sigma=\sum_{i, j, F} \int d x_{1} d x_{2} d \hat{\Phi}_{F} w_{i j F} \frac{d \hat{\sigma}_{i j \rightarrow F}^{\text {selected }}(\hat{s} ; m, \alpha)}{d \hat{\Phi}_{F}}
$$

Here, $d \hat{\sigma}_{i j \rightarrow F}^{\text {selected }}\left(\hat{s} ; m, \alpha_{s}\right)$ is the cross section for the base subprocess at the c.m. energy squared, masses, and couplings. The $w_{i j F}$ is the weight factor which takes into account the PDFs, CKM matrix $V_{\mathrm{CKM}}$, and decay factors of $W / Z$ bosons:

$$
w_{i j F}=f_{i}^{1}\left(x_{1}, Q^{2}\right) f_{j}^{2}\left(x_{2}, Q^{2}\right)\left|V_{\mathrm{CKM}}\right|^{2 K},
$$

where $K$ is the number of $W$ boson in the process.

The GR@PPA code can be obtained from its web page 14]. Outline of GR@PPA is explained in the following; however, much details should be found in the original articles [14, 15]. A cross section can be calculated in the following steps.

(1) Particles and vertices in theory

First, a theoretical model is supplied in the form of particle content, parameters, and interaction vertices derived from a model Lagrangian. In this work, the model is the standard model of strong and electro-weak interactions. The model information is supplied as model files.

(2) Input for particles and kinematics

Initial- and final-state particles as well as reaction kinematics need to be supplied for calculating cross sections. The kinematical conditions are c.m. energy, kinematical cuts for jets and final-state particles, hard scales for the running coupling constant and PDFs, renormalization and factorization scales, and the others. They are supplied in the files grcpar.F and upinit.F as instructed in Table II of Ref. [14]. The kinematical cuts should be supplied as they were used in the CDF analysis.

(3) Diagram generation

From the supplied model information on the particles and vertices, Feynman diagrams are automatically generated by using mathematical techniques of graph theory. Here, a node corresponds to a vertex or an external particle, and an edge does to a propagator or a connection between a vertex and an external particle. First, vertices are generated for a given condition of a process. Then, the vertices are connected by considering all the possible processes to satisfy obvious conservations such as electric charge and fermion number. Finally, particles are assigned to the propagators.

(4) Matrix-element generation

The generated Feynman diagrams are automatically calculated by using spinors and gamma matrices in a numerical way. Based on the Feynmandiagram configuration, the code calls the model libraries in (1) for calculating each amplitude. Here, propagators are expressed by bi-linear forms of wave functions, Dirac spinors for fermions, and polarization vectors for spin-1 bosons.

(5) Monte Carlo integrals

Finally, the phase-space integrals of Eq. (2) have been calculated by the Monte-Carlo method BASES. The parameter NCALL is the number of sampling points in integration grids, and it should be large enough to obtain convergent numerical results. The recommended values of NCALL are supplied in the subroutine grcpar.F, but they may be changed according to kinematical conditions.

In the published GR@PPA code, the single-top production is not supplied, so that a separate code is created. In this work, the single-top and $t \bar{t}$ production processes are calculated, and resulting distributions are shown as top events.

Since there are many processes which contribute to the $p+\bar{p} \rightarrow e^{ \pm}\left(\mu^{ \pm}\right)+2$ jets $+\not_{T}$, we cannot show all of them here. Only typical processes are shown. For example, one of $W+$ dijet processes is shown in Fig. 1, where $W^{-}$is created in an intermediate stage to produce an electron and an anti-neutrino in the final state, and two jets are produced by a $q \bar{q}$ pair from a hard gluon. Several more processes are shown in Appendix $\mathrm{A}$ for providing an idea of other contributions. For getting information on all the processes, one may look at figure files created by running the GR@PPA. In the same way, typical contributions of $Z+$ dijet, top, $W W$, and $Z W$ processes are shown in Figs. 2, 3, 4, and 5, respectively.

In our work, elementary partonic cross sections are calculated by the GR@PPA. Subsequent parton branching and final fragmentation into hadrons are not included. They could be calculated, for example, by using the event generator PYTHIA [16]. It is the purpose of this work to show gross properties of strange-quark effects simply by calculating the hard process part. In future, if much detailed comparisons become necessary with the CDF and D0 experimental data, they needs to be considered. This work is the first step toward such an approach.

\section{B. Applied kinematical conditions in GR@PPA}

In obtaining the cross section, various kinematical cuts are applied. They are the same as the ones used in the 


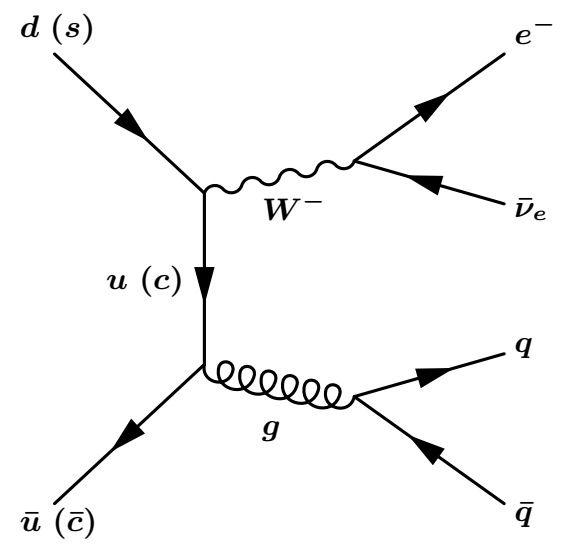

FIG. 1: Typical $W+$ dijet process. The notation $q$ indicates a quark $u, d, s$, or $c$.

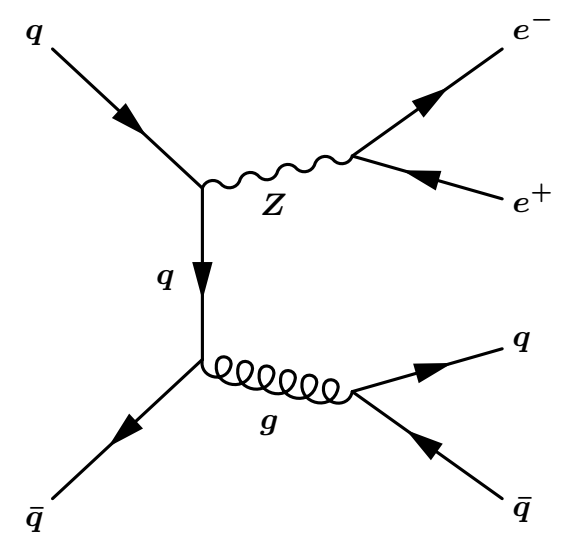

FIG. 2: Typical $Z+$ dijet process.

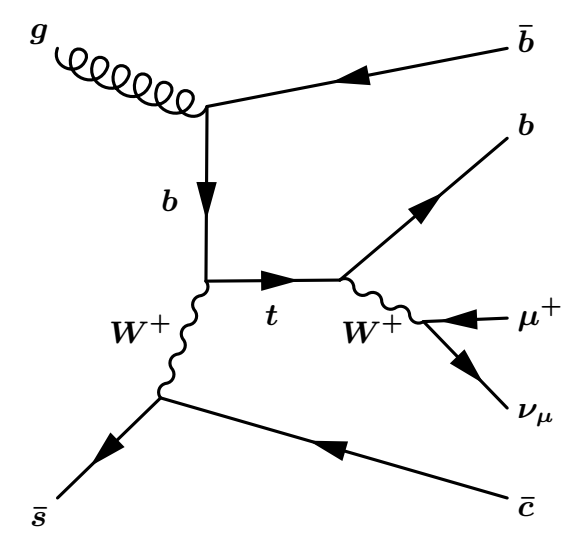

FIG. 3: Typical top-production process.

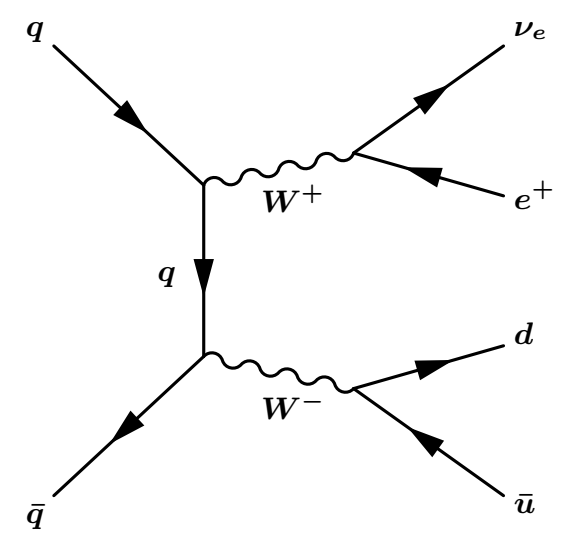

FIG. 4: Typical $W W$ process.

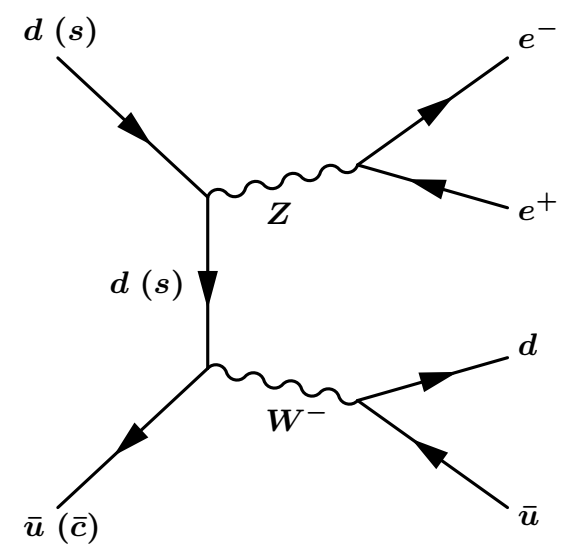

FIG. 5: Typical $Z W$ process.
CDF analysis [1]. In order to search for the signal $p+$ $\bar{p} \rightarrow W W / Z W \rightarrow \ell \nu_{\ell}+$ dijets, events with (two energetic jets $)+\left(\right.$ one high- $p_{T}$ electron or muon $)+\left(\right.$ missing $\left.E_{T}\right)$ are selected. The high- $p_{T}$ lepton from $W$ needs to be accompanied by missing transverse energy $\left(\mathbb{E}_{T}\right)$ for a non-interacting neutrino.

The momentum cut $p_{T}^{\ell}>20 \mathrm{GeV}$ is applied for removing QCD backgrounds from semi-leptonic decays of hadrons and $\left|\eta^{\ell}\right|<1.0$ is due to detector ranges. For selecting the leptonic $W$ candidate, a cut is also applied for the missing $E_{T}$ as $E_{T}>25 \mathrm{GeV}$ in order to remove the QCD backgrounds. Furthermore, the transverse-mass cut $M_{T}^{W}>30 \mathrm{GeV}$ also reduces the QCD backgrounds. The cone radius $\Delta R=\sqrt{(\Delta \phi)^{2}+(\Delta \eta)^{2}}=0.4$ is used for the jet definition with the cuts for each jet as $E_{T}^{\text {jet }}>30$ $\mathrm{GeV}$ and $\eta_{j}<2.4$. Additional cut conditions are applied as $\left|\Delta \eta_{j j}\right|<2.5, \Delta \phi_{\text {ET }_{T} j_{1}}>0.4, \Delta R_{j \ell}>0.52$, and $p_{T j j}>40 \mathrm{GeV}$ for rejecting back-to-back events, multi-jet (fake missing $\mathscr{E}_{T}$ ) events, semi-leptonic decays 
of hadrons, and for better agreement between data and simulation, respectively. Gaussian smearing effects are taken into account for $W$ and $Z$ by assigning an effective width of $15 \mathrm{GeV}$ in the dijet-mass distribution.

\section{STRANGE-QUARK DISTRIBUTION FUNCTION}

\section{A. Definition and experimental information}

The quark distribution function $q_{i}(x)$ with flavor $i$ is defined by the amplitude for the nucleon $(N)$ to emit the quark $i$ and then to absorb it at a separated light-cone coordinate $y^{-}$[17

$$
\begin{array}{r}
q_{i}(x)=\int \frac{d y^{-}}{4 \pi} e^{-i x p^{+} y^{-}}\langle N(p)| \bar{\psi}_{i}\left(0^{+}, y^{-}, \overrightarrow{0}_{\perp}\right) \\
\times \gamma^{+} \wp \psi_{i}\left(0^{+}, 0^{-}, \overrightarrow{0}_{\perp}\right)|N(p)\rangle,
\end{array}
$$

where $\wp$ is the path-ordered $(P)$ link to make the expression gauge invariant:

$$
\wp=P \exp \left\{-i g \int_{0}^{y^{-}} d z^{-} A_{a}^{+}\left(0^{+}, z^{-}, \overrightarrow{0}_{\perp}\right) t_{a}\right\} .
$$

The $a^{ \pm}$indicates the light-cone coordinates $a^{ \pm}=\left(a^{0} \pm\right.$ $\left.a^{3}\right) / \sqrt{2}$, and $\vec{a}_{\perp}$ is the two-dimensional transverse coordinate. The $\psi$ and $A$ are quark and gluon fields, respectively, and $t_{a}$ is the generator of $S U(3)$ group and it is given by the Gell-Mann matrix $\lambda_{a}$ as $t_{a}=\lambda_{a} / 2$.

The PDFs can be calculated in nonperturbative theoretical methods such as lattice QCD, bag model, chiral soliton model, and so on. However, obtained PDFs are not accurate enough to supply precise cross sections, so that global analyses of world data have been done for obtaining the optimum PDFs.

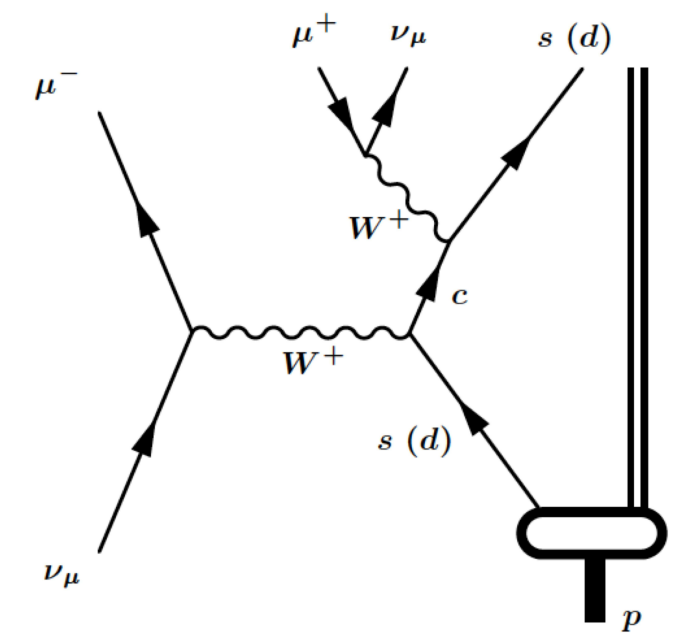

FIG. 6: Strange-quark distribution in neutrino-induced opposite-sign dimuon production process $\left(\nu_{\mu}+p \rightarrow \mu^{-} \mu^{+}+\right.$ $X)$.
As explained in Sec. I, the important PDF to be investigated is the strange-quark distribution $s(x)$ in connection with the anomalous CDF result. The strange distribution has been determined from dimuon measurements in neutrino DIS $\left(\nu_{\mu}+p \rightarrow \mu^{-} \mu^{+}+X\right)$ [10] as shown in Fig. 6. The dimuon production occurs from an initial strange (or down) quark to produce an intermediate charm quark which decays into $W^{+}$and $s$ (or $d$ ). From measurements, the strange-quark-moment ratio to the light-antiquark one

$$
\kappa=\frac{\int d x x\left[s\left(x, Q^{2}\right)+\bar{s}\left(x, Q^{2}\right)\right]}{\int d x x\left[\bar{u}\left(x, Q^{2}\right)+\bar{d}\left(x, Q^{2}\right)\right]},
$$

was obtained. This ratio varies depending on experiments, but it is in the range of $0.3 \lesssim \kappa \lesssim 0.5[10]$.

This neutrino process has been the only constraint for determining the strange distribution $s(x)$ in global PDF analyses. However, the HERMES collaboration recently indicated that the distribution $s(x)$ could be much different from the current one [12] by semi-inclusive kaon production in Fig. 7. The shaded blob in the figure indicates a fragmentation process, which is described by the fragmentation functions (FFs). It should be noted that the uncertainties of the FFs are very large for the kaon as shown in Ref. [13]. For example, such an uncertainty effect is illustrated in determining the polarized strangequark distribution $\Delta s(x)$ from data sets including semiinclusive measurements [18]. In any case, considering the large differences between $s(x)$ of the HERMES and the conventional one, we think that the strange distribution could have much larger uncertainties than the ones suggested by most PDF analyses [11].

\section{B. Modifications of strange-quark distribution}

We discuss modifications of the strange-quark distribution $s(x)+\bar{s}(x)$ obtained in a typical global analysis.

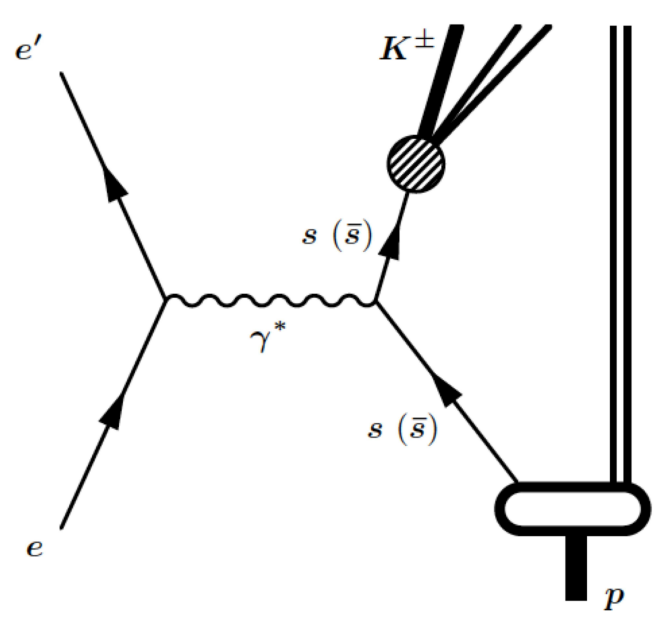

FIG. 7: Semi-inclusive kaon production in charged-lepton deep inelastic scattering $\left(e+p \rightarrow e^{\prime}+K+X\right)$. 
We define the weight function or modification function $w_{i}\left(x, Q^{2}\right)$ for the strange-quark distribution by

$$
\begin{aligned}
& {\left[s\left(x, Q^{2}\right)+\bar{s}\left(x, Q^{2}\right)\right]_{i}} \\
& \quad=w_{i}\left(x, Q^{2}\right)\left[s\left(x, Q^{2}\right)+\bar{s}\left(x, Q^{2}\right)\right]_{\mathrm{PDF} \text { set }},
\end{aligned}
$$

at any $Q^{2}$, where perturbative $\mathrm{QCD}$ can be applied. Here, the CTEQ6L1 distributions [19] are used for the PDF set of the global analysis because they have been used in the GR@PPA code. Namely, the function $w_{i}$ indicates a modification from the $s+\bar{s}$ distribution of the CTEQ global analysis. The index $i$ indicates a modification type ( $i=1$ or 2 ) explained in the following.

As the first possible choice, the weight function is determined from measurements of the strange-quark distribution $s(x)+\bar{s}(x)$ by the HERMES collaboration. The average $Q^{2}$ of the HERMES data is $2.5 \mathrm{GeV}^{2}$, so that we define $Q_{0}^{2}=2.5 \mathrm{GeV}^{2}$ as the scale in providing the initial PDFs including the modification. The following hyperbolic-tangent function $w_{1}$ is assigned for the modification by looking at the differences between the CTEQ6L1 distribution and the HERMES data in Fig. 8

$$
w_{1}\left(x, Q_{0}^{2}\right)=1-\tanh \left(\frac{x-x_{0}}{\Delta x}\right) .
$$

The HERMES $x(s+\bar{s})$ distribution is much larger than the CTEQ6L1 at $x<0.06$ and it is much smaller at $x>0.09$. The parameter $x_{0}$ indicates the cross-over point, and $\Delta x$ does the width of the modification curve. These two parameters are determined so as to fit the HERMES data, and obtained values are listed in Table [1. The obtained weight function $w_{1}\left(x, Q_{0}^{2}\right)$ is shown in Fig. 8, and it explain the data well except for slight deviations at $x \sim 0.3$.

In addition to the function $w_{1}$, we consider the following one:

$$
\begin{aligned}
w_{2}\left(x, Q_{0}^{2}\right)= & \frac{1}{2}\left[1+\tanh \left(\frac{x-x_{0}}{\Delta x}\right)\right] \\
& \times \frac{\left[\bar{u}\left(x, Q_{0}^{2}\right)+\bar{d}\left(x, Q_{0}^{2}\right)\right]_{\mathrm{PDF} \text { set }}}{\left[s\left(x, Q_{0}^{2}\right)+\bar{s}\left(x, Q_{0}^{2}\right)\right]_{\mathrm{PDF} \text { set }}} .
\end{aligned}
$$

This function $w_{2}(x)$ has the opposite functional form to $w_{1}(x)$ in the sense that the small- $x$ part is suppressed and the large- $x$ distribution is enhanced to reach to the $\bar{u}+\bar{d}$ distribution. This functions $w_{2} \cdot x(s+\bar{s})$ is shown in Fig. 8 in comparison with the HERMES data, $w_{1} \cdot x(s+\bar{s})$, and $x(\bar{u}+\bar{d})$. Although $w_{2}\left(x, Q_{0}^{2}\right)$ is not supported by

TABLE I: Parameter values in the weight functions of Eqs. (8), (9), and (10) at $Q_{0}^{2}=2.5 \mathrm{GeV}^{2}$ and $Q^{2}=2 M_{W}^{2}$.

\begin{tabular}{c|cccc}
\hline Function & $x_{0}$ & $\Delta x$ & $c_{0}$ & $c_{1}$ \\
\hline$w_{1}\left(x, Q_{0}^{2}\right)$ & 0.0796 & 0.0253 & - & - \\
$w_{2}\left(x, Q_{0}^{2}\right)$ & 0.0796 & 0.0253 & - & - \\
$w_{1}\left(x, Q^{2}\right)$ & 0.0768 & 0.0408 & 0.802 & -0.475 \\
$w_{2}\left(x, Q^{2}\right)$ & 0.0727 & 0.0220 & 1.589 & 0.417 \\
\hline
\end{tabular}

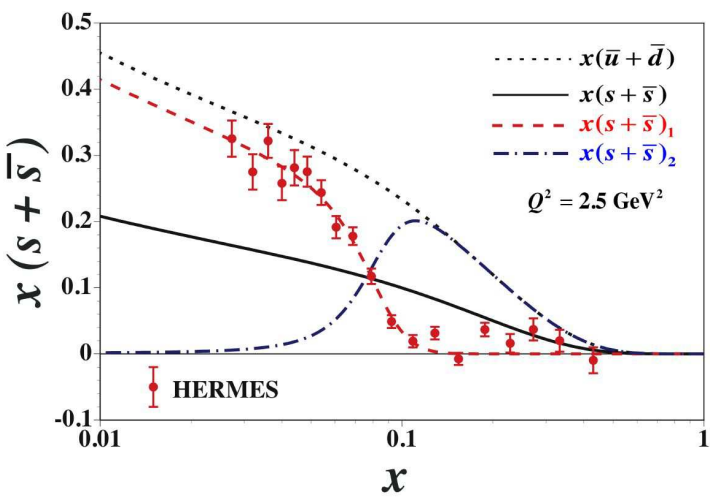

FIG. 8: $x(s+\bar{s})$ distribution functions are compared with HERMES data 12] and $x(\bar{u}+\bar{d})$. The CTEQ6L1 distribution $x(s+\bar{s})$ [19] is shown by the solid curve, and the modified distributions by $w_{1}\left(x, Q_{0}^{2}\right)$ and $w_{2}\left(x, Q_{0}^{2}\right)$ are shown by the dashed and dash-dotted curves, respectively. The weight functions $w_{1}\left(x, Q_{0}^{2}\right)$ and $w_{2}\left(x, Q_{0}^{2}\right)$ are explained in the main text. For comparison, the $x(\bar{u}+\bar{d})$ distribution is shown by the dotted curve.

the HERMES data, we simply consider it as a trial function to investigate sensitivity of the CDF result on the strange distribution. The strange distribution is roughly constrained by the neutrino measurements; however, its $x$-dependent functional form is not determined. In particular, the large- $x$ region is not explored experimentally. As there is an issue of a significant intrinsic charm distribution at large $x$ 20, 21, there may be an enhancement in the strange at large $x$ 222]. From Fig. 8 , the small- $x$ distribution may seem to be extraordinarily small. However, if the function $w_{2}$ is evolved to the average $Q^{2}(\sim 20$ $\mathrm{GeV}^{2}$ ) of CCFR and NuTeV measurements, the suppression is not very large as it becomes clear in Sec. IIIC. It should be also noted that these functions $w_{1}$ and $w_{2}$ roughly correspond to the bounds of the $s(x)$ uncertainty in the CT10 distribution [11].

\section{C. $Q^{2}$ evolution}

The hard scale $Q^{2}$ in providing the PDFs for the CDF measurements is much larger than $Q_{0}^{2}=2.5 \mathrm{GeV}^{2}$, where the HERMES data are provided for the $s+\bar{s}$ distribution, so that the strange-quark distribution needs to be evolved. As a typical hard scale in calculating the CDF dijet cross sections, we take $Q^{2}=2 M_{W}^{2}$ by considering the dijet mass $m_{j j} \sim 100 \mathrm{GeV}$ and the boson (W and Z) masses. The standard DGLAP (Dokshitzer-GribovLipatov-Altarelli-Parisi) equations are used for the $Q^{2}$ evolution by using the code of Ref. 23]. In the case without the modification $(w=1)$, we checked that the evolved distribution is numerically consistent with the CTEQ one. The evolved strange distributions are shown in Fig. 9, The strange quarks are copiously produced especially at small $x$ due to $Q^{2}$ evolution, so that the distributions become large at small $x$. Therefore, the 


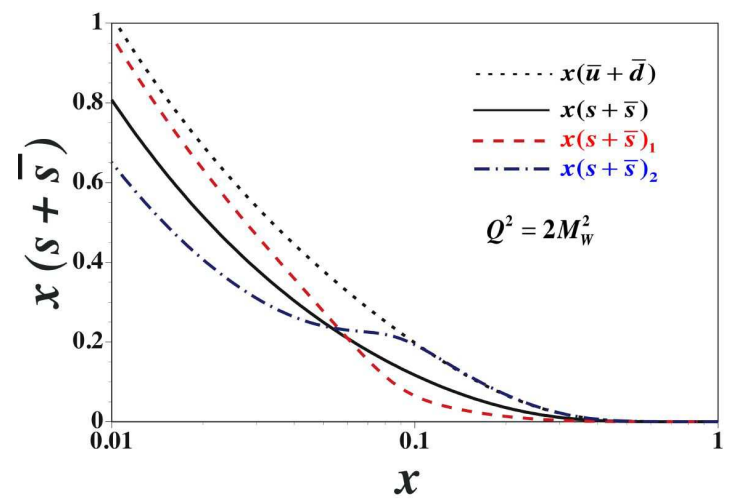

FIG. 9: $Q^{2}$ evolved $x(s+\bar{s})$ distribution functions are shown. The CTEQ6L1 distribution [19] and the modified distributions by $w_{1}\left(x, Q_{0}^{2}\right)$ and $w_{2}\left(x, Q_{0}^{2}\right)$ are evolved to $Q^{2}=2 M_{W}^{2}$. For comparison, the $x(\bar{u}+\bar{d})$ distribution is shown at $Q^{2}=$ $2 M_{W}^{2}$ by the dotted curve.

differences between $(s+\bar{s})_{\mathrm{PDF} \text { set }},(s+\bar{s})_{1}$, and $(s+\bar{s})_{2}$ become smaller than the original ones at $Q_{0}^{2}$. In comparison, the large- $x(>0.1)$ distributions are not so significantly modified by the $Q^{2}$ evolution.

In order to clearly show the evolution of the weight functions, we divide the evolved strange distributions $s_{i}\left(x, Q^{2}\right)$ by the CTEQL1 distribution to calculate the weight function $w_{i}\left(x, Q^{2}\right)$ as given in Eq. (7) at any $Q^{2}$. In Fig. 10, three $Q^{2}$ values are taken for showing the $Q^{2}$ evolution of the weight functions $w_{1}$ and $w_{2}$. They are $Q^{2}=2.5 \mathrm{GeV}^{2}, 20 \mathrm{GeV}^{2}$, and $2 M_{W}^{2}$ for the initial HERMES scale, NuTeV scale, and CDF-jet scale, respectively. Although the modifications are large at $Q^{2}=2.5 \mathrm{GeV}^{2}$ and it is in the range $0<w_{1,2} \lesssim 2$, they become smaller as $Q^{2}$ increases. At the neutrino DIS scale of $Q^{2}=20 \mathrm{GeV}^{2}$, the modifications becomes smaller $\left(0.5 \lesssim w_{1,2} \lesssim 1.5\right)$ at small $x(<0.08)$. In comparison with $\bar{u}+\bar{d}$ at $Q^{2}=20 \mathrm{GeV}^{2}$, the strange to light-antiquark ratio is $0.23 \lesssim(s+\bar{s}) /(\bar{u}+\bar{d}) \lesssim 0.68$, which is not very different from the neutrino measure-

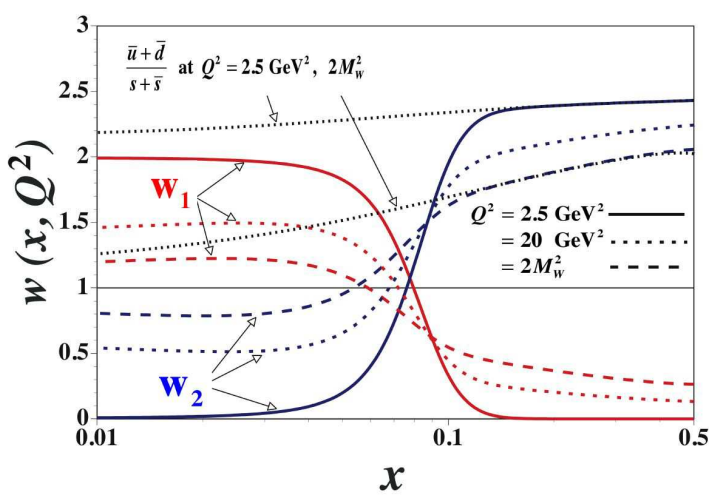

FIG. 10: The weight functions $w_{1}\left(x, Q^{2}\right)$ and $w_{2}\left(x, Q^{2}\right)$ are shown at $Q^{2}=2.5 \mathrm{GeV}^{2}, 20 \mathrm{GeV}^{2}$, and $2 M_{W}^{2}$ by the solid, dotted, and dashed curves, respectively. For comparison, the ratios $(s+\bar{s}) /(\bar{u}+\bar{d})$ are shown at $Q^{2}=2.5 \mathrm{GeV}^{2}$ and $2 M_{W}^{2}$. ments [10] although one should note that the neutrinoexperimental ratio of Eq. (6) is for the second moments. At $Q^{2}=2 M_{W}^{2}$, the modifications become much smaller and they are in the range $0.8 \lesssim w_{1,2} \lesssim 1.2$ at small $x$ $(<0.08)$. The modifications also become smaller with increasing $Q^{2}$ at large $x(>0.1)$; however, $Q^{2}$ variations are not as large as the ones at small $x$.

For including the modifications into the event generator, the evolved weight functions are fitted by the functions

$$
\begin{aligned}
w_{1}\left(x, Q^{2}\right)= & c_{0}-c_{1} \tanh \left(\frac{x-x_{0}}{\Delta x}\right), \\
w_{2}\left(x, Q^{2}\right)= & \frac{1}{2}\left[c_{0}+c_{1} \tanh \left(\frac{x-x_{0}}{\Delta x}\right)\right] \\
& \times \frac{\left[\bar{u}\left(x, Q^{2}\right)+\bar{d}\left(x, Q^{2}\right)\right]_{\mathrm{PDF} \text { set }}}{\left[s\left(x, Q^{2}\right)+\bar{s}\left(x, Q^{2}\right)\right]_{\mathrm{PDF} \text { set }}}
\end{aligned}
$$

where $c_{0}, c_{1}, x_{0}$, and $\Delta x$ are the parameters determined by the fitting. Obtained parameters are listed in Table [1. Since it takes time to calculate the cross sections by the Monte Carlo integrals in the GR@PPA, these fitted functions are used instead of calculating the $Q^{2}$ evolution within the Monte Carlo code.

\section{Comments on recent PDFs and theoretical models}

We comment on the strange-quark distribution in recent global analyses. Until a few years ago, the distribution $s(x)$ is simply assumed to be proportional to $[\bar{u}(x)+\bar{d}(x)] / 2$ together with the assumption $s(x)=\bar{s}(x)$. However, more flexible parametrizations were recently investigated in some analyses by allowing independent $x$ dependence for $s(x)$. Then, the uncertainty of the determined $s(x)$ became large in general. The size of the uncertainty of $s(x)$ depends on analysis groups. A large uncertainty was suggested in the CT10 PDF set for the strange-quark distribution as shown in Fig. 12 of Ref. 11] although uncertainties of other analysis groups are smaller. The CT10 indicates a large uncertainty range $0<w\left(x, Q^{2}\right)<2$ at the scale $Q^{2}=4 \mathrm{GeV}^{2}$ depending on the $x$ region. This uncertainty range roughly corresponds to the functions $w_{1,2}\left(x, Q^{2}\right)$ in Fig. 10. Therefore, although the modifications seem to be very large especially at $Q^{2}=2.5 \mathrm{GeV}^{2}$ in Figs. 8 and 10 , they are allowed by the current experimental data. As it is explained in recent global-analysis articles [11], that $Z$ and $W$ production cross sections at LHC are sensitive to the strangequark distribution, so that this issue may be solved in future LHC measurements 24].

Theoretically, the strange-quark distribution stems from perturbative and non-perturbative mechanisms. The perturbative creation of $s \bar{s}$ pairs is described by the DGLAP $Q^{2}$ evolution equations. The non-perturbative contributions are calculated by phenomenological models 
such as meson clouds, chiral soliton, and so on; however, obtained distributions inevitably depend on the used model. The lattice QCD method has been making progress; however, it is not at the stage to provide the detailed $x$ dependent functional form. A few lowmoments of the PDFs have been obtained so far in the lattice QCD [25].

As a nonperturbative effect on the PDFs, an intrinsic charm distribution has been often discussed. The charm distribution $c(x)$ is usually considered to be created by a perturbative mechanism by the gluon splitting $g \rightarrow$ $c \bar{c}$; however, there could exist the intrinsic charm in the proton according to the light-cone Fock space picture [20]

$$
|p>=| u u d>+\cdots+\mid u u d c \bar{c}>+\cdots .
$$

In addition, a meson-cloud picture also suggests the intrinsic charm distribution by $p(u u d) \rightarrow \bar{D}^{0}(u \bar{c}) \Lambda_{c}^{+}(u d c)$, $p(u u d) J / \psi(c \bar{c})[26]$. In the same way, the nonperturbative strange-quark distribution could exist by considering the strange-quark content in the proton as [22]

$$
|p>=| u u d>+\cdots+\mid u u d s \bar{s}>+\cdots .
$$

It is known that the intrinsic charm distribution appears as a bump in the large- $x$ region as shown in Figs. 2 and 3 of Ref. 21]. In the same way, the strange-quark distribution could have a distribution in the large- $x$ region, which may be related to the CDF anomaly. These nonperturbative studies have been done for light antiquark $(\bar{u}$ and $\bar{d})$ distributions [7, 27] and for the strange distribution [22] as well as for the charm [20]. In this article, we do not step into the details of these mechanisms and their comparisons with the CDF anomaly.

In future, it is important to investigate the details of the theoretical models and the lattice QCD for the strange-quark distribution, and then their connection to the CDF anomaly. Since there is no established theory for the strange-quark distribution, we simply vary the CTEQ strange distribution to the softer one of the HERMES collaboration and to the harder one as explained in Sec. IIIB.

\section{LEPTON-PLUS-DIJET CROSS SECTIONS}

Using the GR@PPA code and the modified strangequark distributions, we calculate each process contribution to the lepton+dijet cross section. In particular, $W+$ dijet, $Z+$ dijet, top, $W W$, and $Z W$ cross sections are calculated. Using the CTEQ6L1 PDFs without the strange modification, we obtain the events as a function of the dijet mass in Fig. 11. We checked that numerical results are consistent with other ones, for example, by using the ALPGEN [28] by calculating these cross sections for Tevatron and LHC kinematics.

The lepton-plus-dijet cross sections are dominated by the $W+$ dijet processes and other contributions are small. The ordinate indicates the event rate in the dijet mass

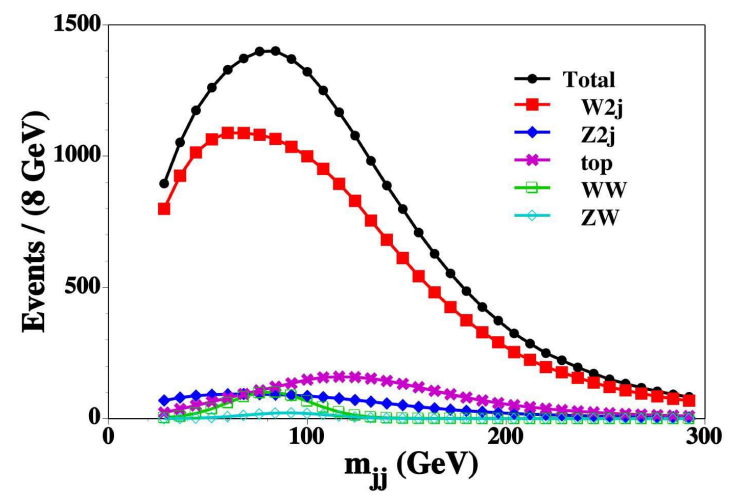

FIG. 11: Contributions to lepton-plus-dijet events in the CDF kinematics. The $W+$ dijet, $Z+$ dijet, top, $W W$, and $Z W$ events and their total are shown. Here, the default strangequark distribution is used without modification.

interval of $8 \mathrm{GeV}$. Since the detector acceptance information is not available for public, and since parton shower and final fragmentations are not included in our calculations, the magnitude of the ordinate cannot be compared with the CDF measurements. Therefore, we may look at overall shapes and each contribution. We find that the overall features agree with the CDF measurements.

Next, we show the strange-quark effects on the cross section. The $Q^{2}$ evolved weight functions $w_{1}\left(x, Q^{2}\right)$ and $w_{2}\left(x, Q^{2}\right)$ are used for calculating the strange-quark distributions in the GR@PPA. Obtained event rates are shown in Fig. 12. It indicates that the cross section increases if the hard strange-quark distribution $s_{2}\left(x, Q^{2}\right)$ is used, and it decreases if the soft distribution $s_{1}\left(x, Q^{2}\right)$ is used. As mentioned in the introduction section, the $x$ region which affects the $\mathrm{CDF}$ result is $x \sim 0.1$. As shown in Fig. 9, the distribution $s(x)$ significantly increases in the hard strange distribution $s_{2}(x)$ and decreases in the soft distribution $s_{1}(x)$ at $x \sim 0.1$. This fact gives rise to the modifications in the lepton+dijet cross sections.

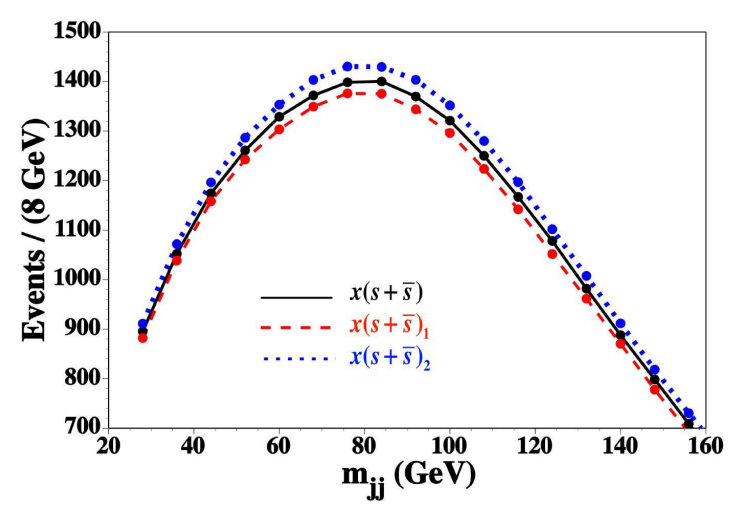

FIG. 12: Effects of the strange-quark distribution on leptonplus-dijet events in the CDF kinematics. Three curves are shown by taking the strange-quark distribution $s(x)$ without modification and the distributions multiplied by $w_{1}\left(x, Q^{2}\right)$ and $w_{2}\left(x, Q^{2}\right)$. 


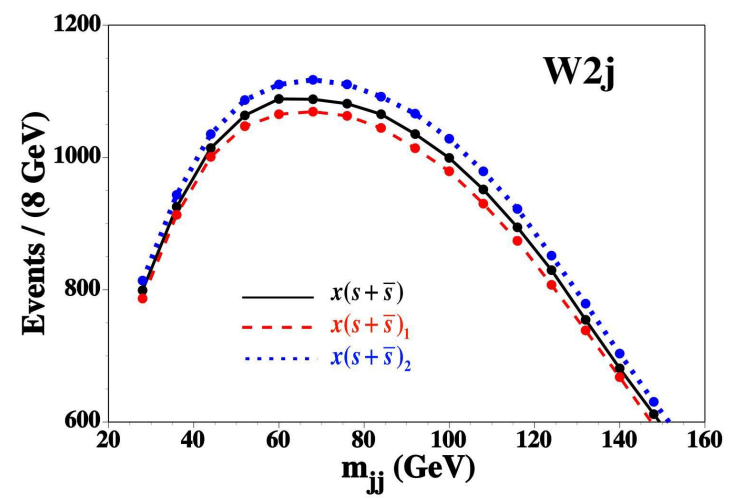

FIG. 13: Effects of the strange-quark distribution on $W+$ dijet events in the CDF kinematics. Three curves are shown by taking the strange-quark distribution $s(x)$ without modification and the distributions multiplied by $w_{1}\left(x, Q^{2}\right)$ and $w_{2}\left(x, Q^{2}\right)$.

It is interesting to find that the distribution shape as the function of the dijet mass becomes wider if the hard strange-quark distribution is used. It has a tendency to partially explain the anomalous CDF excess, although a sharp CDF-like bump is difficult to be obtained within modifications of PDFs. However, because the CDF finding is in the shoulder region of the cross section, a slight shift in the cross-section shape may explain the CDF excess if they accumulate enough data to obtain accurate cross sections.

We mentioned a possible bump structure of $s(x)$ in Sec. IIID According to our experience on the numerical estimates, it is not easy to explain the sharp CDF peak by a bump in $s(x)$ at medium $x$, even if it exists, because cross sections are obtained by integrating the PDFs over a certain region of $x$, more specifically over a certain rapidity range. In addition, the $Q^{2}$ evolution smears out the bump.

The modification effects are calculated for each process. We found that there are sizable effects on the $W+$ dijet and $Z+$ dijet cross sections from the strangequark modification. However, the effects are very small in top, $W W$, and $Z W$ production processes. Since the magnitude of the cross section is largest in $W+$ dijet, we show the modification effect on this process in Fig. 13 . Because the lepton+dijet cross section is dominated by the $W+$ dijet process, the overall modification effects are similar to the ones in the total event rates in Fig. 12 The hard strange-quark distribution increases the cross section and shifts the shape toward the larger dijet-mass region, whereas the soft distribution has opposite effects.

Next, the same $W+$ dijet cross sections are calculated for the LHC kinematics of $14 \mathrm{TeV}$ c.m. energy. Two replacements in the c.m. energy and initial state, $\sqrt{s}=$ $1.96 \mathrm{TeV} \rightarrow 14 \mathrm{TeV}$ and $p \bar{p} \rightarrow p p$, are simply done in the GR@PPA code for the calculation. The other cut conditions are kept the same simply for finding kinematical effects. The obtained results are shown in Fig. 14. We notice large differences from the the event rates of the CDF kinematics in Fig. 13] The cross section decreases

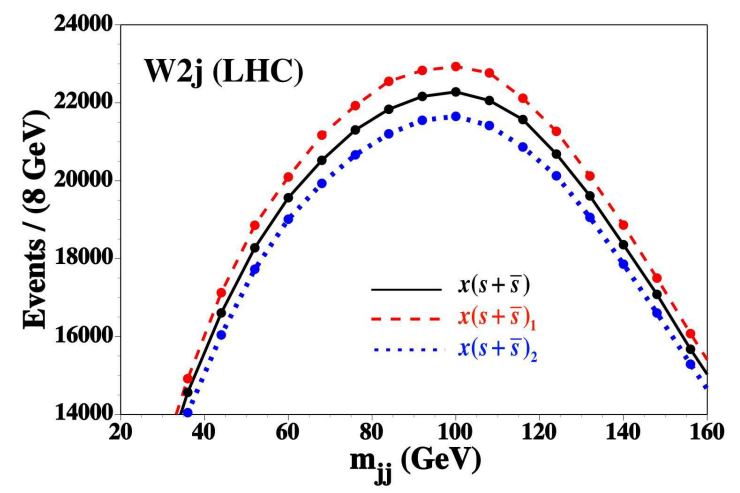

FIG. 14: Effects of the strange-quark distribution on $W+$ dijet events in the LHC kinematics. The notations are the same in Fig. 13 .

(increases) by the hard (soft) strange-quark distribution and the shape becomes narrower (wider). These tendencies are opposite to the CDF ones in Fig. 13. This is simply caused by kinematical effects. Because the c.m. energy is much larger in the LHC, the process is sensitive to the smaller- $x$ region, particularly at $x \sim 0.02$. Here, the hard distribution $s_{2}(x)$ becomes smaller than the standard one without the modification at $x \sim 0.02$, and the soft one $s_{1}(x)$ becomes larger as shown in Fig. 9 These differences produce the opposite strange-modification effects to the ones of the CDF kinematics.

These results indicates an interesting conclusion that the CDF analysis should be carefully done by considering the uncertainty of the strange-quark distribution. Furthermore, the strange-quark distribution can be tested by future LHC experiments. In particular, the extraordinary HERMES result on $s(x)$ can be tested by both Tevatron and LHC measurements on vector-boson productions 24]. It is important to note that Tevatron and LHC probe different $x$ regions of the strange-quark distribution. There is still a possibility that the CDF anomaly could be explained within the standard model in terms of the PDF modification if the peak in the dijet-mass spectrum is not too sharp.

We did not step into the actual calculations based on some theoretical models. We simply explored effects due to the modifications of the strange-quark distribution on the lepton+dijet cross sections. In future, it should be interesting to theoretically investigate the strange-quark momentum distribution by hadron models and possibly by lattice QCD. We leave them for our future projects.

\section{SUMMARY}

We investigated a possibility that the anomalous CDF result in the lepton+dijet cross section can be explained within the standard model by modifications of the parton distribution functions. In particular, the effects of the strange-quark distribution have been calculated by using the event generator GR@PPA. The strange-quark distri- 
bution $s(x)$ has been thought to be determined by the opposite-sign dimuon measurements in neutrino deepinelastic scattering. However, it became obvious by the HERMES collaboration that its momentum distribution, namely the $x$ dependence, has not been reliably determined at this stage because the much softer distribution was suggested by their data.

In this work, two modifications were considered for the strange-quark distribution. One is the soft distribution suggested by the HERMES collaboration, and another is the hard distribution. The modified distributions were evolved to the ones at the scale $Q^{2}=2 M_{W}^{2}$. The hard distribution increases the lepton+dijet cross section and also spread the cross-section shape toward the large dijetmass region. It tends to partially explain the CDF excess. The sizable strange-quark-modification effects were obtained for the subprocesses of $W+$ dijet and $Z+$ dijet productions, whereas the effects are very small for the subprocesses of top, $W W$, and $Z W$ productions. We found that the PDFs in the region of $x \sim 0.1$ affect the CDF lepton+dijet cross sections.

The modification effects were also calculated for the LHC kinematics, and the interesting results were obtained in the sense that opposite effects are found to the Tevatron case. The hard distribution decreases the cross section and shrinks the dijet-mass spectrum, which is opposite to the ones in the Tevatron kinematics. This is caused by the fact that the PDFs in the region $x \sim 0.02$ affect the lepton+dijet cross section in the LHC kinematics. These studies indicate that the $x$ dependence of the strange-quark distribution can be investigated by the $W$ and $Z$ production measurements in both Tevatron and LHC. These two-facility experiments probe different $x$ regions of the strange-quark distribution.

There are still large uncertainties originating from the strange-quark distribution. If the CDF anomalous peak is not too sharp, there is still a room to explain the excess within the standard model in terms of the PDFs without introducing any new physics beyond the standard model.

\section{Acknowledgments}

The authors thank Y. Miyachi for communications on the HERMES experiment and S. Odaka for suggestions on the GRAPPA usage. This work was partially supported by a Grant-in-Aid for Scientific Research on Priority Areas "Elucidation of New Hadrons with a Variety of Flavors (E01: 21105006)" from the ministry of Education, Culture, Sports, Science and Technology of Japan.

\section{Appendix A: An example of $W+$ dijet processes}

There are too many processes, which contribute to the $W+$ dijet cross section, to show all of them. The figure files to show all the processes are automatically generated by running the GR@PPA code. The reader may look at the processes in postscript files created by running the GR@PPA. One of the files is shown in Fig. 15]

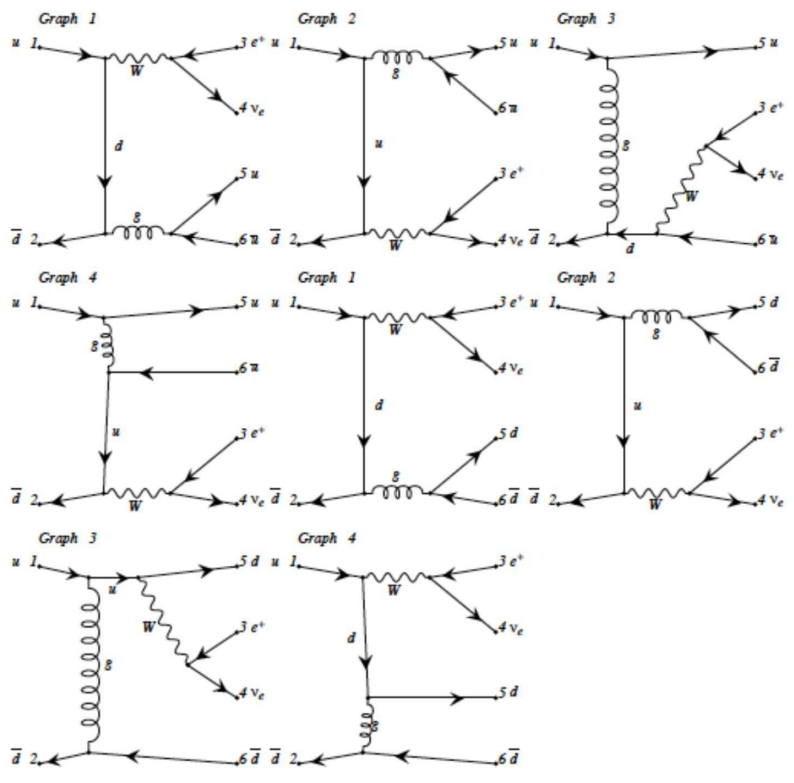

FIG. 15: Some of $W+$ dijet processes are shown by taking a figure file created by running the GR@PPA code. These are just a part of all the calculated processes for the $W+$ dijet events.
[1] T. Aaltonen et al. (CDF Collaboration), Phys. Rev. Lett. 106, 171801 (2011); V. Cavaliere, Ph. D. thesis, Siena University, Dec. 2010, http://lss.fnal.gov/archive/thesis /fermilab-thesis-2010-51.shtml; G. Punzi, talk at the 23rd Rencontres de Blois, Particle Physics and Cosmology, Château Royal de Blois, France, May 29 - June 3, 2011.

[2] V. M. Abazov et al. (D0 Collaboration), Phys. Rev. Lett. 107, 011804 (2011).

[3] F. Abe et al. (CDF), Phys. Rev. Lett. 77, 438 (1996).

[4] H. L. Lai et al. (CTEQ Collaboration), Phys. Rev. D 55,
1280 (1997); J. Pumplin et al., JHEP 07, 012 (2002).

[5] A complete list of theoretical works should be found by the CDF citation list, http://www.slac.stanford.edu /spires/find/hep?c=PRLTA,106,171801. There are too many theoretical papers to list them here, so that we quote some of them in the following. M. R. Buckley, D. Hooper, J. Kopp, and E. Neil, Phys. Rev. D 83, 115013 (2011); F. Yu, Phys. Rev. D 83, 094028 (2011); E. J. Eichten, K. Lane, and A. Martin, Phys. Rev. Lett. 106, 251803 (2011). There are some works within the standard model: X.-G. He and B.-Q. Ma, arXiv:1104.1894 
[hep-ph]; Z. Sullivan and A. Menon, Phys. Rev. D 83, 091504 (2011). T. Plehn and M. Takeuchi, J. Phys. G 38, 095006 (2011); J. M. Campbell, A. Martin, and C. Williams, Phys. Rev. D 84, 036005 (2011).

[6] I. Schienbein et al., Phys. Rev. D 77, 054013 (2008); 80, 094004 (2009); K. Kovarik et al., Phys. Rev. Lett. 106, 122301 (2011); M. Hirai, S. Kumano, and K. Saito, AIP Conf. Proc. 1189, 269 (2009); M. Hirai, S. Kumano, and T.-H. Nagai, Phys. Rev. C 76, 065207 (2007).

[7] S. Kumano, Phys. Rept. 303, 183 (1998); G. T. Garvey and J.-C. Peng, Prog. Part. Nucl. Phys. 47, 203 (2001).

[8] See http://p25ext.lanl.gov/e866/e866.html for the Fermilab-E906 experiment.

[9] See http://j-parc.jp/index-e.html for the J-PARC project. S. Kumano, J. Phys. Conf .Ser. 312, 032005 (2011).

[10] A. Kayis-Topaksu et al. (CHORAS Collaboration), Nucl. Phys. B 798, 1 (2008); U. Dore, arXiv:1103.4572 [hepex].

[11] CTEQ6.6: P. M. Nadolsky et al., Phys. Rev. D 78, 013004 (2008); CT10: H.-L. Lai et al., Phys. Rev. D 82, 074024 (2010); MSTW2008: A. D. Martin et al., Eur. Phys. J. C 63, 189 (2009); ABKM-2010: S. Alekhin et al., Phys. Rev. D 81, 014032 (2010); JR-2009: P. JimenezDelgado and E. Reya, Phys. Rev. D 79, 074023 (2009); NNPDF2.0: R. D. Ball et al., Nucl. Phys. B 838, 136 (2010); HERAPDF1.0: F. D. Aaron et al., JHEP 01, 109 (2010).

[12] A. Airapetian et al. (HERMES Collaboration), Phys. Lett. B 666, 446 (2008).

[13] M. Hirai, S. Kumano, T.-H. Nagai, and K. Sudoh, Phys. Rev. D 75, 094009 (2007).

[14] S. Odaka and Y. Kurihara, arXiv:1107.4467 [hep-ph]; S. Tsuno, T. Kaneko, Y. Kurihara, S. Odaka, and K. Kato, Comput. Phys. Commun. 175, 665 (2006). See http://atlas.kek.jp/physics/nlo-wg/grappa.html for obtaining the GR@PPA code and its explanation.

[15] See http://www-sc.kek.jp/ for the GRACE. J. Fujimoto et al., Comput. Phys. Commun. 153, 106 (2003); G. Be- langer et al., Phys. Rept. 430, 117 (2006).

[16] See http://home.thep.lu.se/ torbjorn/Pythia.html for the event generator PYTHIA.

[17] G. Sterman et al. (CTEQ Collaboration), Rev. Mod. Phys. 67, 157 (1995).

[18] E. Leader, A. V. Sidorov, and D. B. Stamenov, Phys. Rev. D 84, 014002 (2011).

[19] J. Pumplin et al., JHEP 07, 012 (2002).

[20] S. J. Brodsky, P. Hoyer, C. Peterson, and N. Sakai, Phys. Lett. B 93, 451 (1980); S. J. Brodsky, C. Peterson, and N. Sakai, Phys. Rev. D 23, 2745 (1981); S. J. Brodsky, A. S. Goldhaber, B. Z. Kopeliovich, and I. Schmidt, Nucl. Phys. B 807, 334 (2009).

[21] J. Pumplin, H. L. Lai, and W. K. Tung, Phys. Rev. D 75, 054029 (2007).

[22] S. J. Brodsky and B.-Q. Ma, Phys. Lett. B 381, 317 (1996); H. Chen, F.-G. Cao, and A.I. Signal, J. Phys. G 37, 105006 (2010) and references therein.

[23] M. Miyama and S. Kumano, Comput. Phys. Commun. 94, 185 (1996).

[24] Strange-quark effects on LHC measurements are discussed in the articles of Ref. [11], for example, in p.12 of CTEQ6.6; pp.14-16 in CT10; pp.238-242 of MSTW2008; pp.14-15 in ABKM-2010.

[25] M. Göckeler et al. (QCDSF Collaboration), Phys. Rev. D 71, 114511 (2005); S. Collins et al. (QCDSF/UKQCD Collaboration), PoS LATTICE2010, 153 (2010); S. Dinter et al. Phys. Lett. B 704, 89 (2011).

[26] F. S. Navarra et al., Phys. Rev. D 54, 842 (1996); S. Paiva et al., Mod. Phys. Lett. A 13, 2715 (1998); W. Melnitchouk and A.W. Thomas, Phys. Lett. B 414, 134 (1997); F. M. Steffens, W. Melnitchouk, and A.W. Thomas, Eur. Phys. J. C 11, 673 (1999).

[27] S. Kumano, Phys. Rev. D 43, 59 (1991); 3067 (1991); S. Kumano and J. T. Londergan, Phys. Rev. D 44, 717 (1991). There are many articles on this topic, and they should be found in Ref. [7].

[28] M. L. Mangano et al., JHEP 07, 001 (2003). 